\title{
RETIFICADOR TRIFÁSICO ISOLADO COM ALTO FATOR DE POTÊNCIA UTILIZANDO O CONVERSOR ZETA NO MODO DE CONDUÇÃO CONTÍNUA
}

\author{
Denizar C. Martins, Márcio M. Casaro e Ivo Barbi \\ Universidade Federal de Santa Catarina \\ Departamento de Engenharia Elétrica - Instituto de Eletrônica de Potência \\ Caixa Postal 5119-88040-970 - Florianópolis-SC-BRAZIL \\ Tel.: (048) 331-9204 - FAX: 48.234-5422 - Email: denizar@inep.ufsc.br
}

\begin{abstract}
Resumo - Este trabalho apresenta a análise de um retificador trifásico isolado para correção do fator potência, empregando o conversor Zeta operando no modo de condução contínua. A estrutura é particularmente simples e robusta. Suas principais características são: emprega um único estágio para processamento de energia, o qual pode operar como abaixador ou elevador de tensão, baixa distorção harmônica da corrente de entrada, e isolação natural. $O$ conversor trabalha com freqüência constante empregando técnica PWM. O princípio de operação, o procedimento de projeto e os resultados obtidos por simulação e experimentais são apresentados.
\end{abstract}

Abstract - This paper presents the analysis of an Isolated Three-Phase Rectifier with high power factor using a Zeta converter operating in continuous conduction mode (CCM). The structure is particularly simple and robust. Its main features are: one power processing stage, which can operate as steep-down or steep-up voltage, lower harmonic distortion of the input current and natural isolation. The converter works with constant frequency and PWM technique. Principle of operation, design procedure and experimental results are presented.

\section{INTRODUÇÃO}

Nos últimos vinte anos o avanço da Eletrônica de Potência tem alcançado níveis surpreendentes, não somente em relação às variações topológicas, como também nas estratégias de comando e controle dos conversores estáticos.

Devido a esse enorme desenvolvimento os conversores estáticos têm sido utilizados nas mais variadas aplicações industriais para baixas e altas potências, constituindo-se nos dias atuais como um dos importantes temas de estudo na área da Engenharia Elétrica.

Apesar desse extraordinário desempenho, o estágio de entrada da maioria dos conversores estáticos emprega uma ponte retificada acoplada a um filtro capacitivo de valor expressivo. A associação desses componentes gera uma carga não-linear que conectada ao sistema de energia elétrica comercial causa distorção na corrente de entrada.

Nas aplicações industriais de elevada potência (acima de $1 \mathrm{~kW}$ ), os sistemas trifásicos de alimentação são geralmente os mais recomendados, onde a conversão CA/CC tem sido dominada por retificadores convencionais a diodos e retificadores controlados a tiristor. A característica não linear da corrente de entrada destes retificadores, conforme já mencionado, cria problemas para a rede comercial de energia elétrica, entre os quais podem ser destacados:

- injeção de elevado conteúdo harmônico na corrente de entrada;

- distorção da tensão de alimentação, devido aos altos picos da corrente de entrada;

- aumento das perdas nas linhas de energia;

- redução do fator de potência;

- necessidade de geração de grandes quantidades de potência reativa;

- diminuição do rendimento da estrutura devido ao elevado valor eficaz da corrente de entrada.

Devido a esses inconvenientes, muitos trabalhos têm sido apresentados pela comunidade científica de eletrônica de potência, a fim de proporcionar a utilização de conversores CA/CC com elevado fator de potência e baixo conteúdo harmônico da corrente de entrada [1]-[12].

Normalmente a correção do fator de potência é obtida empregando-se conversores com característica de entrada como fonte de corrente. Para sistemas trifásicos esse procedimento é quase uma regra.

Tomando como exemplo o conversor Boost [1], que atualmente é um dos conversores mais cogitados para correção do fator de potência, pode-se observar as seguintes características: conversor elevador de tensão, não é naturalmente isolado e cada fase apresenta um indutor Boost.

Tentando superar essas limitações básicas, o presente artigo está propondo a aplicação de um retificador trifásico isolado empregando o conversor CC-CC Zeta em condução contínua para a correção do fator de potência. As principais características dessa topologia são: conversor abaixador ou elevador de tensão, isolação natural, apresenta um único estágio de processamento de energia, saída com característica de fonte de corrente o que facilita a associação de vários módulos em paralelo, robustez e simplicidade na estrutura de potência e no circuito de controle.

Além das características já mencionadas é importante salientar que a estrutura proposta permite, sem muita dificuldade e com custos relativamente baixos, elevar o fator de potência de circuitos convencionais, que estão sendo utilizados na indústria, com a simples inclusão do módulo Zeta entre o retificador trifásico convencional e a carga, conforme pode ser visto na Figura 1. 


\section{PRINCÍPIO DE OPERAÇÃO}

\section{A. Circuito Proposto}

O circuito proposto é mostrado na Figura 1.

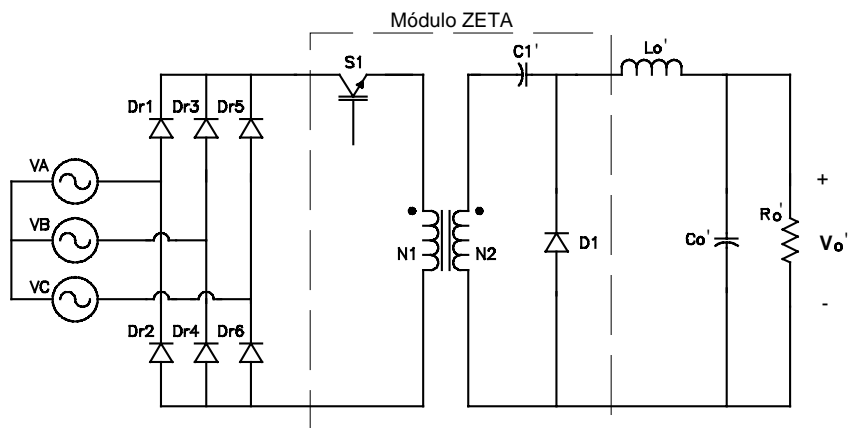

Figura 1: Circuito proposto.

As Figuras 2 e 3 apresentam o circuito equivalente com todos os parâmetros referidos ao lado primário do transformador, onde:

$$
\begin{gathered}
\mathrm{V}_{\mathrm{o}}=\left(\mathrm{N}_{1} / \mathrm{N}_{2}\right) \cdot \mathrm{V}_{\mathrm{o}}{ }^{\prime} ; \mathrm{C}_{\mathrm{o}}=\left(\mathrm{N}_{2} / \mathrm{N}_{1}\right)^{2} \cdot \mathrm{C}_{\mathrm{o}}{ }^{\prime} ; \mathrm{C}_{1}=\left(\mathrm{N}_{2} / \mathrm{N}_{1}\right)^{2} \cdot \mathrm{C}_{1}{ }^{\prime} \\
\mathrm{L}_{\mathrm{o}}=\left(\mathrm{N}_{1} / \mathrm{N}_{2}\right)^{2} \cdot \mathrm{L}_{\mathrm{o}}{ }^{\prime} ; \mathrm{R}_{\mathrm{o}}=\left(\mathrm{N}_{1} / \mathrm{N}_{2}\right)^{2} \cdot \mathrm{R}_{\mathrm{o}}{ }^{\prime}
\end{gathered}
$$

\section{B. Etapas de Operação}

Com o objetivo de simplificar a análise, as seguintes considerações são feitas:

- o circuito opera em regime permanente;

- os semicondutores são considerados ideais;

- o transformador é representado por sua indutância de magnetização referida ao primário;

- a capacitância $C_{0}$ possui valor suficientemente elevado para manter a tensão de saída $\mathrm{V}_{\mathrm{o}}$ constante;

- a tensão da rede é considerada constante durante um período de chaveamento.

O conversor Zeta operando em condução contínua apresenta duas etapas de funcionamento:

$1^{\mathrm{a}}$ ETAPA $\left(\mathrm{t}_{0}, \mathrm{t}_{1}\right)$ - Figura 2: No instante $\mathrm{t}_{0}$ a chave $\mathrm{S}_{1}$ é fechada e conduz a corrente $i_{S 1}$, que cresce linearmente. A fonte de alimentação transfere energia para o indutor magnetizante $L_{m}$ e o capacitor $C_{1}$ transfere energia para a indutância $\mathrm{L}_{\mathrm{o}}$. Durante esta etapa o diodo $\mathrm{D}_{1}$ se mantém bloqueado com tensão reversa igual a $-\left(\mathrm{V}_{\mathrm{in}}+\mathrm{V}_{\mathrm{o}}\right)$. As correntes $i_{\text {Lm }}$ e $i_{\text {Lo }}$ crescem linearmente. As tensões $V_{C o}$ e $V_{C 1}$ são consideradas constantes e igual a $\mathrm{V}_{\mathrm{o}}$.

$2^{\mathrm{a}}$ ETAPA $\left(\mathrm{t}_{1}, \mathrm{t}_{2}\right)$ - Figura 3: Em $\mathrm{t}=\mathrm{t}_{1}$, a chave $\mathrm{S}_{1}$ é bloqueada e o diodo $\mathrm{D}_{1}$ entra em condução, permitindo que os indutores $\mathrm{L}_{\mathrm{m}}$ e $\mathrm{L}_{\mathrm{o}}$ transfiram suas energias para os capacitores $C_{1}$ e $C_{0}$, respectivamente. As correntes $i_{L m}$ e $i_{L o}$ decrescem linearmente. A tensão sobre a chave $S_{1}$ é igual a $\left(\mathrm{V}_{\text {in }}+\mathrm{V}_{\mathrm{o}}\right)$.
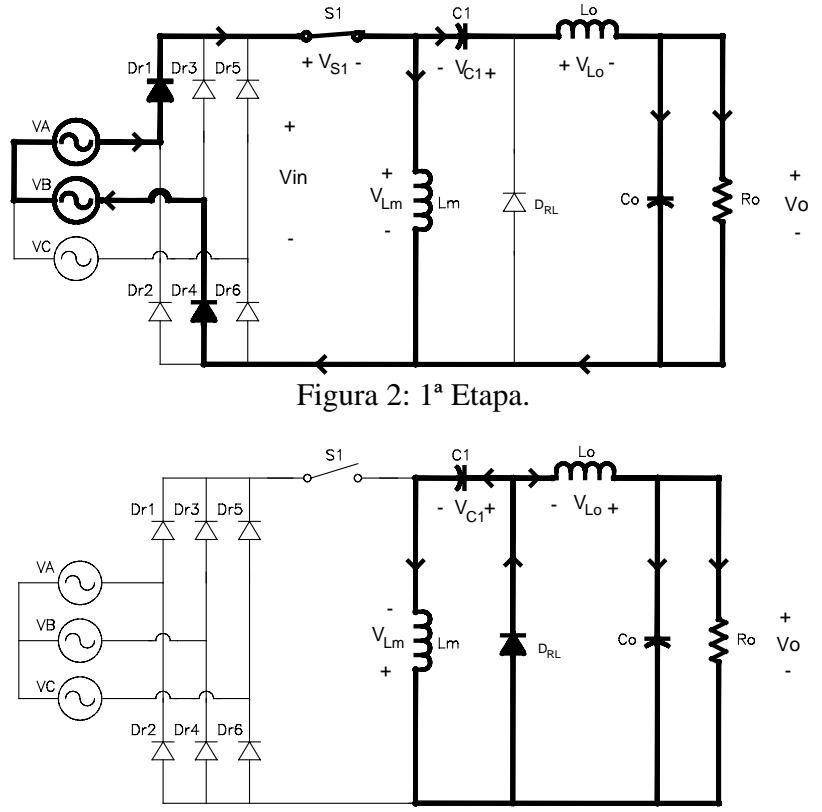

Figura 3: $2^{\text {a }}$ Etapa.

As principais formas de ondas estão apresentadas na Figura 4.

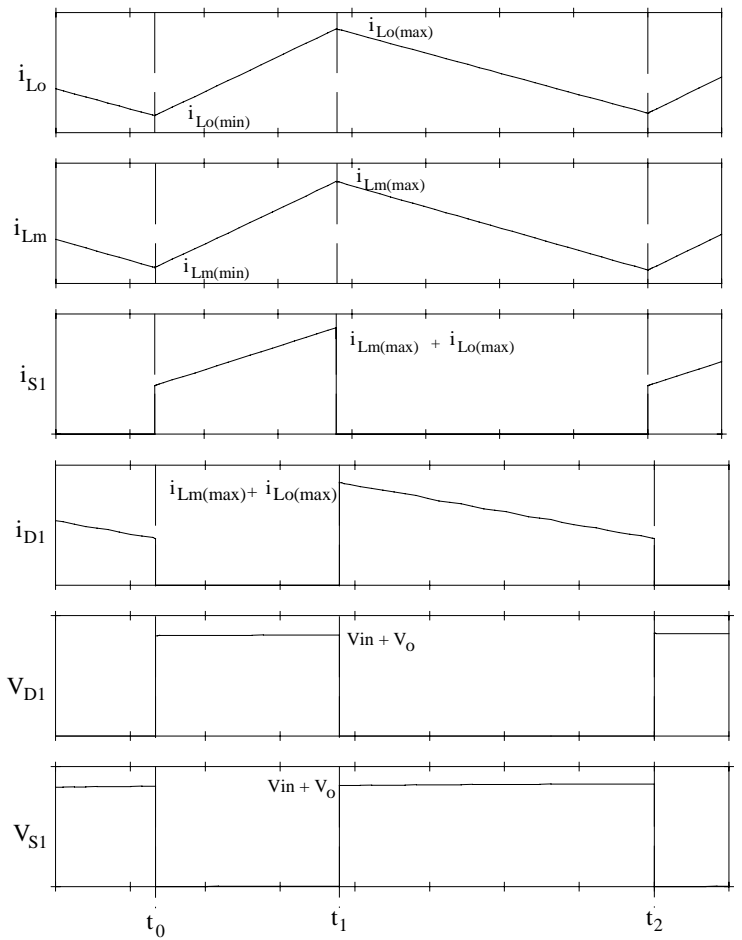

Figura 4: Principais formas de ondas.

C. Análise Quantitativa

Definindo: $\mathrm{t}_{\mathrm{f}}=\mathrm{t}_{1}-\mathrm{t}_{0}$

$$
\begin{aligned}
& \mathrm{t}_{\mathrm{a}}=\mathrm{t}_{2}-\mathrm{t}_{1}=\mathrm{T}_{\mathrm{S}}-\mathrm{t}_{\mathrm{f}} \\
& \mathrm{T}_{\mathrm{S}}=1 / \mathrm{f}_{\mathrm{S}} \\
& \mathrm{D}=\mathrm{t}_{\mathrm{f}} / \mathrm{T}_{\mathrm{S}} \\
& \mathrm{V}_{\text {in }}=\sqrt{3} \mathrm{~V}_{\mathrm{p}} \operatorname{sen}(\omega \mathrm{t}) ; \text { para } \omega \mathrm{t} \text { variando de } \pi / 3
\end{aligned}
$$

até $2 \pi / 3$. 
1) $1^{a}$ Etapa $\left(0 \leq t \leq t_{f}\right)$

Condições iniciais: $\mathrm{i}_{\mathrm{Lm}(\mathrm{t}=0)}=\mathrm{i}_{\mathrm{Lm}(\min )}$

$$
\begin{aligned}
& \mathrm{i}_{\mathrm{Lo}(\mathrm{t}=0)}=\mathrm{i}_{\mathrm{Lo}(\min )} \\
& \mathrm{V}_{\mathrm{Lm}(\mathrm{t})}=\mathrm{V}_{\mathrm{Lo}(\mathrm{t})}=\mathrm{V}_{\text {in }}
\end{aligned}
$$

A partir do circuito equivalente referente a esta etapa chega-se às seguintes equações:

$$
\begin{gathered}
\mathrm{V}_{\mathrm{Lm}(\mathrm{t})}=\mathrm{L}_{\mathrm{m}} \frac{\mathrm{di}_{\mathrm{Lm}(\mathrm{t})}}{\mathrm{dt}} \\
\mathrm{V}_{\mathrm{Lm}(\mathrm{t})}=\mathrm{V}_{\mathrm{in}}=\sqrt{3} \mathrm{~V}_{\mathrm{p}} \operatorname{sen}(\omega \mathrm{t})
\end{gathered}
$$

Trabalhando as equações (1) e (2) obtém-se a expressão da corrente no indutor magnetizante:

$$
\mathrm{i}_{\mathrm{Lm}(\mathrm{t})}=\sqrt{3} \frac{\mathrm{V}_{\mathrm{p}}}{\mathrm{L}_{\mathrm{m}}} \mathrm{t} \cdot \operatorname{sen}(\omega \mathrm{t})+\mathrm{i}_{\mathrm{Lm}(\min )}
$$

Para a indutância de saída $\mathrm{L}_{\mathrm{o}}$ tem-se:

$$
\begin{gathered}
\mathrm{V}_{\mathrm{Lo}(\mathrm{t})}=\mathrm{L}_{\mathrm{o}} \frac{\mathrm{di}_{\operatorname{Lo}(\mathrm{t})}}{\mathrm{dt}} \\
\mathrm{V}_{\mathrm{Lo}(\mathrm{t})}=\mathrm{V}_{\text {in }}
\end{gathered}
$$

Desse modo, seguindo o mesmo procedimento anterior, obtém-se:

$$
\mathrm{i}_{\mathrm{Lo}(\mathrm{t})}=\sqrt{3} \frac{\mathrm{V}_{\mathrm{p}}}{\mathrm{L}_{\mathrm{o}}} \mathrm{t} \cdot \operatorname{sen}(\omega \mathrm{t})+\mathrm{i}_{\mathrm{Lo}(\min )}
$$

A corrente na chave $S_{1}$ é dada pela soma das correntes nas indutâncias $\mathrm{L}_{\mathrm{m}}$ e $\mathrm{L}_{\mathrm{o}}$, resultando em:

$\mathrm{i}_{\mathrm{S} 1(\mathrm{t})}=\mathrm{i}_{\mathrm{Lm}(\mathrm{t})}+\mathrm{i}_{\mathrm{Lo}(\mathrm{t})}=\left(\frac{1}{\mathrm{~L}_{\mathrm{m}}}+\frac{1}{\mathrm{~L}_{\mathrm{o}}}\right) \sqrt{3} \cdot \mathrm{V}_{\mathrm{p}} \cdot \mathrm{t} \cdot \operatorname{sen}(\omega \cdot \mathrm{t})+\mathrm{i}_{\mathrm{Lm}(\min )}+\mathrm{i}_{\mathrm{Lo}(\min )}$

Definindo:

$$
\frac{1}{\mathrm{~L}_{\mathrm{eq}}}=\frac{1}{\mathrm{~L}_{\mathrm{m}}}+\frac{1}{\mathrm{~L}_{\mathrm{o}}}
$$

Então.

$$
\mathrm{i}_{\mathrm{S} 1(\mathrm{t})}=\sqrt{3} \frac{\mathrm{V}_{\mathrm{p}}}{\mathrm{L}_{\mathrm{eq}}} \mathrm{t} \cdot \operatorname{sen}(\omega \mathrm{t})+\mathrm{i}_{\mathrm{Lm}(\min )}+\mathrm{i}_{\mathrm{Lo}(\min )}
$$

2) $2^{a}$ Etapa $\left(0 \leq t \leq t_{a}\right)$

Condições iniciais: $\mathrm{i}_{\mathrm{Lm}(\mathrm{t}=0)}=\mathrm{i}_{\mathrm{Lm}(\max )}$

$$
\begin{aligned}
& \mathrm{i}_{\mathrm{Lo}(\mathrm{t}=0)}=\mathrm{i}_{\mathrm{Lo}(\max )} \\
& \mathrm{V}_{\mathrm{Lm}(\mathrm{t})}=\mathrm{V}_{\mathrm{Lo}(\mathrm{t})}=\mathrm{V}_{\mathrm{o}}
\end{aligned}
$$

A exemplo da $1^{\mathrm{a}}$ Etapa, a partir do circuito equivalente chega-se às seguintes equações:

$$
\begin{gathered}
\mathrm{V}_{\mathrm{Lm}(\mathrm{t})}=\mathrm{L}_{\mathrm{m}} \frac{\mathrm{di}_{\mathrm{Lm}(\mathrm{t})}}{\mathrm{dt}} \\
\mathrm{V}_{\mathrm{Lm}(\mathrm{t})}=-\mathrm{V}_{\mathrm{o}}
\end{gathered}
$$

Através das equações (10) e (11) chega-se à expressão da corrente no indutor magnetizante:

$$
\mathrm{i}_{\mathrm{Lm}(\mathrm{t})}=\mathrm{i}_{\mathrm{Lm}(\max )}-\frac{\mathrm{V}_{\mathrm{O}}}{\mathrm{L}_{\mathrm{m}}} \mathrm{t}
$$

onde:

$$
\mathrm{i}_{\mathrm{Lm}(\max )}=\sqrt{3} \frac{\mathrm{V}_{\mathrm{p}}}{\mathrm{L}_{\mathrm{m}}} \mathrm{t}_{\mathrm{f}} \cdot \operatorname{sen}(\omega \mathrm{t})+\mathrm{i}_{\mathrm{Lm}(\min )}
$$

é obtida a partir da equação (3), fazendo $t=t_{f}$.

Substituindo (13) em (12) resulta:

$$
\mathrm{i}_{\mathrm{Lm}(\mathrm{t})}=\frac{\sqrt{3} \mathrm{~V}_{\mathrm{p}} \mathrm{t}_{\mathrm{f}}}{\mathrm{L}_{\mathrm{m}}} \operatorname{sen}(\omega \mathrm{t})-\frac{\mathrm{V}_{\mathrm{O}}}{\mathrm{L}_{\mathrm{m}}} \mathrm{t}+\mathrm{i}_{\mathrm{Lm}(\min )}
$$

Para a indutância de saída $\mathrm{L}_{\mathrm{o}}$ tem-se:

$$
\begin{gathered}
\mathrm{V}_{\mathrm{Lo}(\mathrm{t})}=\mathrm{L}_{\mathrm{o}} \frac{\mathrm{di}_{\operatorname{Lo}(\mathrm{t})}}{\mathrm{dt}} \\
\mathrm{V}_{\mathrm{Lo}(\mathrm{t})}=-\mathrm{V}_{\mathrm{o}}
\end{gathered}
$$

Substituindo a equação (16) em (15) e resolvendo a equação diferencial resultante, obtém-se:

$$
\mathrm{i}_{\mathrm{Lo}(\mathrm{t})}=\mathrm{i}_{\mathrm{Lo}(\max )}-\frac{\mathrm{V}_{\mathrm{o}}}{\mathrm{L}_{\mathrm{o}}} \cdot \mathrm{t}
$$

onde:

$$
\mathrm{i}_{\mathrm{Lo}(\max )}=\frac{\sqrt{3} \mathrm{~V}_{\mathrm{p}}}{\mathrm{L}_{\mathrm{o}}} \mathrm{t}_{\mathrm{f}} \cdot \operatorname{sen}(\omega \mathrm{t})+\mathrm{i}_{\mathrm{Lo}(\min )}
$$

é obtida a partir da equação (6), fazendo $\mathrm{t}=\mathrm{t}_{\mathrm{f}}$.

Assim, $\mathrm{i}_{\mathrm{Lo}(\mathrm{t})}$ resulta em:

$$
\mathrm{i}_{\mathrm{Lo}(\mathrm{t})}=\frac{\sqrt{3} \mathrm{~V}_{\mathrm{p}}}{\mathrm{L}_{\mathrm{o}}} \mathrm{t}_{\mathrm{f}} \cdot \operatorname{sen}(\omega \mathrm{t})-\frac{\mathrm{V}_{\mathrm{o}}}{\mathrm{L}_{\mathrm{o}}} \mathrm{t}+\mathrm{i}_{\mathrm{Lo}(\min )}
$$

A corrente no diodo $\mathrm{D}_{1}$ é dada pela soma das correntes nos indutores $\mathrm{L}_{\mathrm{m}}$ e $\mathrm{L}_{\mathrm{o}}$. Logo:

$\mathrm{i}_{\operatorname{Dl}(\mathrm{t})}=\mathrm{i}_{\mathrm{Lm}(\mathrm{t})}+\mathrm{i}_{\mathrm{Lo}(\mathrm{t})}=\frac{\sqrt{3} \mathrm{~V}_{\mathrm{p}} \mathrm{t}_{\mathrm{f}}}{\mathrm{L}_{\mathrm{eq}}} \cdot \operatorname{sen}(\omega \mathrm{t})-\frac{\mathrm{V}_{\mathrm{o}}}{\mathrm{L}} \mathrm{t}+\mathrm{i}_{\mathrm{Lm}(\min )}+\mathrm{i}_{\mathrm{Lo}(\min )}$

Retomando a equação (19), é possível afirmar que para $\mathrm{t}=$ $\mathrm{t}_{\mathrm{a}} \Rightarrow \mathrm{i}_{\mathrm{Lo}}=\mathrm{i}_{\mathrm{Lo}(\min )}$. Dessa forma obtém-se:

$$
\mathrm{t}_{\mathrm{a}}=\frac{\sqrt{3} \mathrm{~V}_{\mathrm{p}} \mathrm{t}_{\mathrm{f}}}{\mathrm{V}_{\mathrm{o}}} \cdot \operatorname{sen}(\omega \mathrm{t})
$$

Já que $t_{a}$ é constante, substitui-se $\sqrt{3} \cdot V_{p} \cdot \operatorname{sen}(\omega \cdot t)=V$ in pelo seu valor médio, dado por:

$$
\mathrm{V}_{\text {in } \text { med }}=\frac{3 \sqrt{3}}{\pi} \mathrm{V}_{\mathrm{p}}
$$

resultando em:

$$
\mathrm{t}_{\mathrm{a}}=\frac{3 \sqrt{3} \mathrm{~V}_{\mathrm{p}}}{\pi \mathrm{V}_{\mathrm{o}}} \mathrm{t}_{\mathrm{f}}
$$

Define-se: 


$$
\alpha=\frac{\sqrt{3} \mathrm{~V}_{\mathrm{p}}}{\mathrm{V}_{\mathrm{o}}}
$$

substituindo (24) em (23), obtém-se:

$$
\mathrm{t}_{\mathrm{a}}=\frac{3 \alpha}{\pi} \mathrm{t}_{\mathrm{f}}
$$

\section{PROCEDIMENTO DE PROJETO E EXEMPLO[13]}

\section{A. Especificações de Entrada}

$\mathrm{V}_{\mathrm{f}}=127 \mathrm{~V}$ (valor eficaz da tensão de entrada/fase)

$\mathrm{P}_{\mathrm{o}}=1,5 \mathrm{~kW}$ (potência de saída)

$\mathrm{V}_{\mathrm{o}}{ }^{\prime}=60 \mathrm{~V}$ (tensão média na carga)

$\mathrm{f}_{\mathrm{R}}=60 \mathrm{~Hz}$ (frequiência da rede de alimentação)

$\mathrm{f}_{\mathrm{S}}=20 \mathrm{kHz}$ (freqüência de chaveamento)

B. Relação de Transformação (a)

A relação de transformação escolhida foi de:

$$
\mathrm{a}=\mathrm{N}_{1} / \mathrm{N}_{2}=2 \quad ; \quad \mathrm{V}_{\mathrm{o}}=120 \mathrm{~V}
$$

\section{Ganho Estático $(G)$ e Razão Cíclica (D)}

O ganho estático é definido pela seguinte expressão:

$$
\mathrm{G}=\frac{\mathrm{V}_{\mathrm{o}}}{\sqrt{3} \cdot \mathrm{V}_{\mathrm{p}}}
$$

sendo $\quad V_{p}=\sqrt{2} \cdot V_{f}=180 V$, resulta: $G=0,385$

Por definição $\alpha=1 / \mathrm{G}$; conseqüentemente: $\alpha=2,597$ A partir da Eq. (25) tem-se:

$$
\mathrm{D}=\frac{1}{1+\frac{3}{\pi} \alpha} \quad ; \quad \mathrm{D}=0,3
$$

D. Corrente de Saída ( $\left.I_{o}\right)$ e Resistência de Carga $\left(R_{o}\right)$ Referidas ao Lado Primário

$$
\mathrm{I}_{\mathrm{o}}=\frac{\mathrm{P}_{\mathrm{O}}}{\mathrm{V}_{\mathrm{o}}}=12,5 \mathrm{~A} \quad ; \quad \mathrm{R}_{\mathrm{o}}=\frac{\mathrm{V}_{\mathrm{o}}}{\mathrm{I}_{\mathrm{o}}}=9,6 \Omega
$$

\section{E. Indutância Equivalente $\left(L_{e q}\right)$}

Deseja-se operação em condução contínua a partir de $10 \%$ da carga, portanto:

$$
\mathrm{R}_{\mathrm{O}(\max )}=\frac{\mathrm{V}_{\mathrm{o}}}{10 \% \cdot \mathrm{I}_{\mathrm{o}}}=\frac{120}{0.1 \cdot 12,5} \therefore \mathrm{R}_{\mathrm{o}(\max )}=96 \Omega
$$

A partir do equacionamento apresentado em [9], obtém-se:

$$
\mathrm{L}_{\mathrm{eq}} \geq \frac{\mathrm{R}_{\mathrm{o}(\max )}(1-\mathrm{D})^{2}}{2 \mathrm{f}_{\mathrm{S}}}
$$

Logo: $\mathrm{L}_{\mathrm{eq}} \geq 1,18 \mathrm{mH}$

$$
\text { Foi escolhido o valor de } \Rightarrow \mathrm{L}_{\mathrm{eq}}=1,20 \mathrm{mH}
$$

\section{F. Indutância de Saída $\left(L_{o}\right)$ e Indutância Magnetizante $\left(L_{m}\right)$}

Admitindo uma ondulação de corrente de $1,25 \mathrm{~A}$ pico a pico (10\%), na corrente de saída, tem-se:

$$
\mathrm{L}_{\mathrm{o}}=\frac{\sqrt{3} \cdot \mathrm{V}_{\mathrm{p}} \cdot \mathrm{D}}{\mathrm{f}_{\mathrm{s}} \cdot \Delta \mathrm{i}_{\mathrm{Lo}}} \quad \Rightarrow \quad \mathrm{L}_{\mathrm{o}}=3,74 \mathrm{mH}
$$

Através da relação apresentada na equação (8), obtém-se a indutância magnetizante $\left(\mathrm{L}_{\mathrm{m}}\right)$, ou seja:

$$
\frac{1}{\mathrm{~L}_{\mathrm{eq}}}=\frac{1}{\mathrm{~L}_{\mathrm{m}}}+\frac{1}{\mathrm{~L}_{\mathrm{o}}} \quad \Rightarrow \quad \mathrm{L}_{\mathrm{m}}=1,77 \mathrm{mH}
$$

G. Capacitância de acoplamento $\left(C_{l}\right)$ e de saída $\left(C_{o}\right)$

Para ambos os capacitores adota-se uma ondulação de tensão de $10 \%$ (12V). Desse modo, a partir do equacionamento desenvolvido na Ref.[13] tem-se:

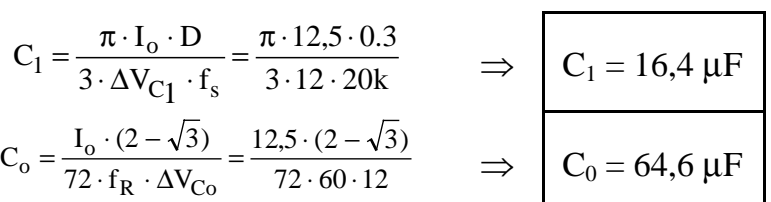

Obs.: Para o projeto do filtro de entrada, foram empregadas as teorias convencionais [14], obtendo-se os seguintes valores:

$\mathrm{C}_{\mathrm{F}}=820 \mathrm{nF}$ (configuração em estrela)

$\mathrm{L}_{\mathrm{F}}=7,72 \mathrm{mH}$

Embora as indutâncias do filtro de entrada resultem num valor relativamente alto, estas podem ser construídas de chapas de ferro silício dando origem a indutores baratos e de baixo volume.

\section{RESULTADOS OBTIDOS POR SIMULAÇÃO}

As Figuras a seguir mostram os principais resultados obtidos através de simulação numérica, utilizando os dados calculados no item anterior.

A Figura 5 apresenta as formas de onda da tensão e da corrente de entrada na situação de carga nominal. Obteve-se nesta situação, um pequeno deslocamento entre a tensão e a fundamental da corrente, na ordem de $-7,862^{\circ}$. Esse deslocamento é devido à indutância de filtragem.

Ainda na situação de carga nominal obteve-se:

$$
\mathrm{TDH}=7,7 \% \text { e } \mathrm{FP}=0,984
$$




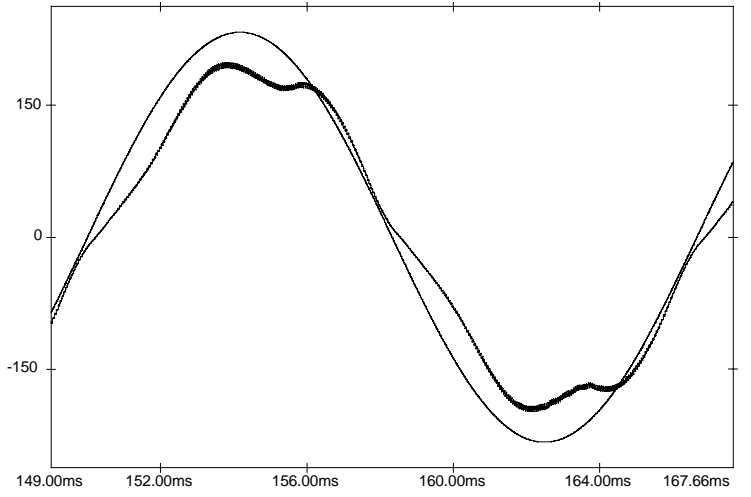

Figura 5 - Tensão e corrente de fase $* 40$.

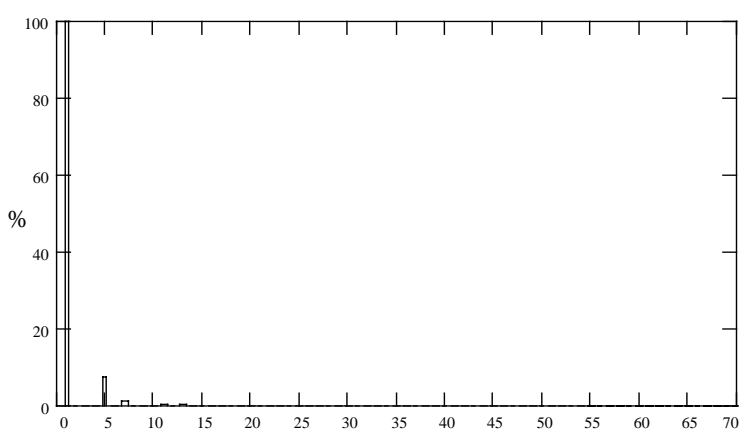

Figura 6 - Análise harmônica da corrente de fase.

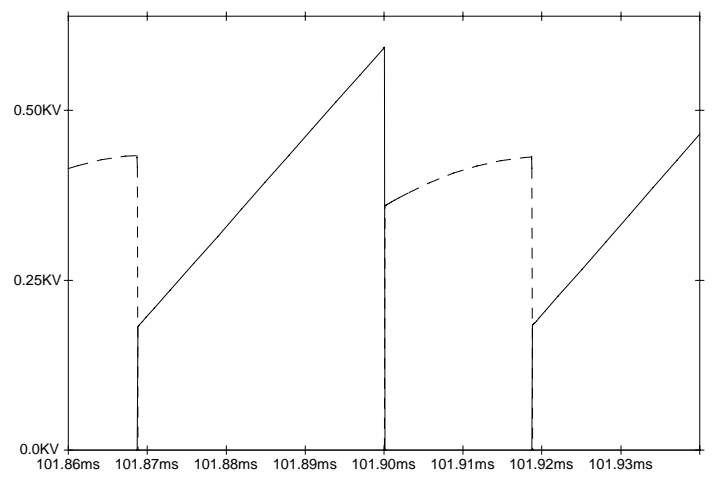

Figura 7 - Tensão na chave $S_{1} \rightarrow$ traço cheio.

Corrente na chave $S_{1}$ multiplicado por $20 \rightarrow$ pontilhado.

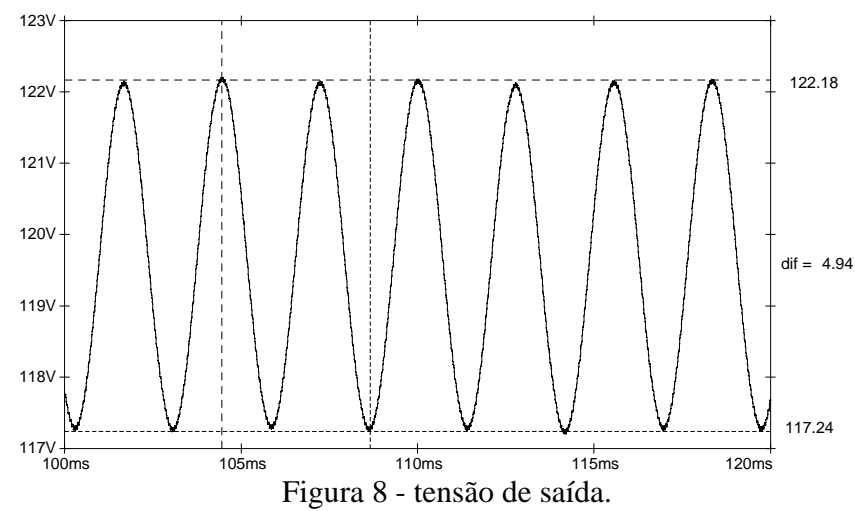

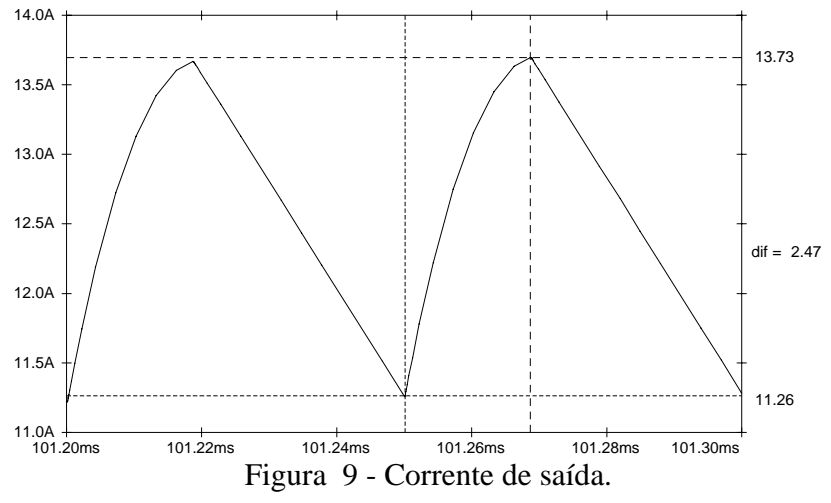

\section{RESULTADOS EXPERIMENTAIS}

Devido a problemas relativos à aquisição de componentes para altas potências, foi implementado um protótipo de laboratório de $1500 \mathrm{~W}$, projetado para operar em CCM até $20 \%$ da carga. Foi utilizado IGBT como chave principal. Outras características do protótipo foram:

$$
\begin{aligned}
\mathrm{V}_{\text {in }}=\sqrt{2} \cdot 127 \cdot \operatorname{sen} \omega \mathrm{t} ; \mathrm{f}_{\mathrm{s}} & =20 \mathrm{kHz} \\
\mathrm{V}_{\mathrm{o}}=60 \mathrm{~V} ; \mathrm{N}_{1} / \mathrm{N}_{2} & =2
\end{aligned}
$$

As principais especificações dos componentes utilizados no protótipo, são dadas a seguir.

* $\quad \mathrm{D}_{1}-\mathrm{D}_{6} \rightarrow$ MUR 3030

* $\quad \mathrm{S}_{1} \rightarrow$ IGBT: IRGBC 40U

* $\mathrm{C}_{\mathrm{F}}=820 \eta \mathrm{F} / 250 \mathrm{~V}$, polipropileno

* $\quad \mathrm{L}_{\mathrm{F}}=7,72 \mathrm{mH}, 249$ espiras

* Transformador de ferrite: EE 65/39, $\mathrm{N}_{1} / \mathrm{N}_{2}=42 / 21$

* $\mathrm{C}_{1}=22 \mu \mathrm{F}$

* $\quad \mathrm{D}_{1}=$ APT $30 \mathrm{D} 60$

* $\mathrm{C}_{\mathrm{o}}=1000 \mu \mathrm{F}$

* $\quad \mathrm{L}_{\mathrm{o}}=3,2 \mathrm{mH} ; 48$ espiras, ferrite EE-42/15

Os resultados experimentais apresentados neste artigo foram obtidos para a condição de plena carga, e com tensão de saída constante igual a $60 \mathrm{~V}$. A Figura 10 apresenta a tensão e a corrente na fase "A". O deslocamento introduzido pela presença do filtro de entrada, é mostrado na Tabela I, para várias percentagens de carga. A análise harmônica é mostrada na Figura 11, onde foi obtida uma distorção harmônica total de $4 \%$ e $6 \%$ respectivamente. Neste caso o fator de potência foi de 0,984 .

Observa-se pela Figura 11 que boa parte da $3^{\mathrm{a}}, 5^{\mathrm{a}}$ e $7^{\mathrm{a}}$ harmônicas da corrente são causadas pela tensão, ou já existem na onda da tensão de fase. Pode-se dizer que se estas harmônicas estiverem em fase o fator de potência estará ainda mais próximo de 1 . 


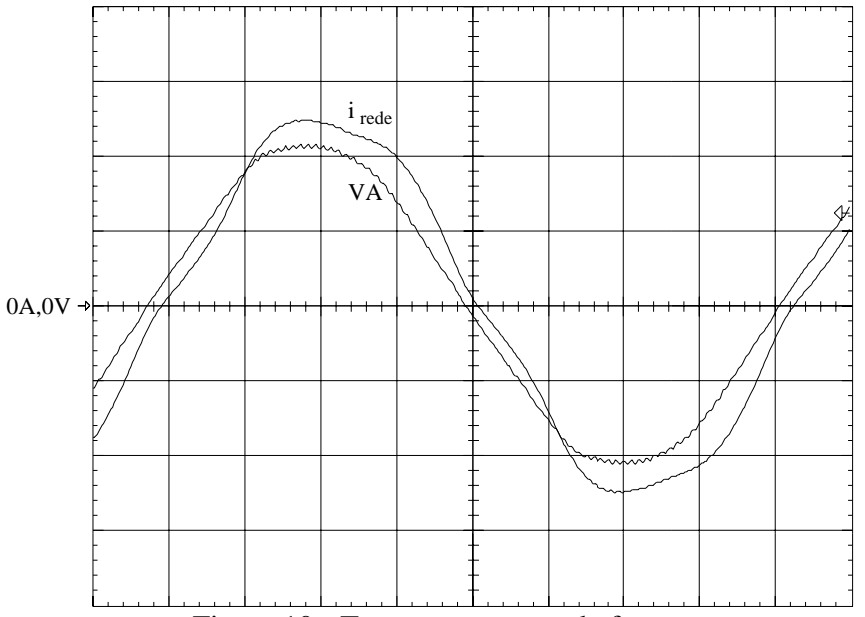

Figura 10 - Tensão e corrente de fase. Escalas: $50 \mathrm{~V} / \mathrm{div}, 4 \mathrm{~A} / \mathrm{div}, 2 \mathrm{~ms} / \mathrm{div}$.
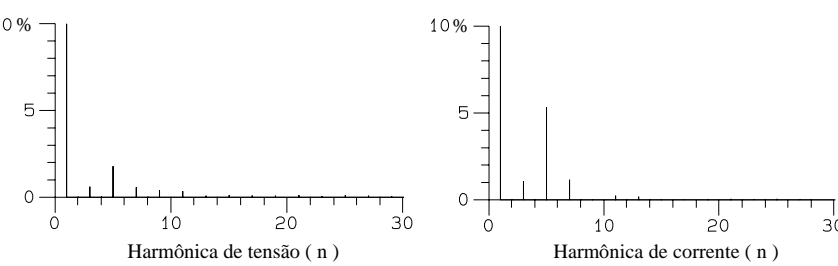

Figura 11 - Análise harmônica da tensão e corrente de fase.

Através de várias aquisições da tensão e corrente de uma das fases, para diferentes valores de carga, e análise harmônica das mesmas, foi possível elaborar a Tabela I, que deu origem as Figuras 12 e 13. As aquisições foram realizadas com a tensão de saída constante em $60 \mathrm{~V}$, com o conversor operando em malha fechada.

TABELA I

\begin{tabular}{|c|c|c|c|}
\hline $\begin{array}{c}\text { carga } \\
\mathbf{\%}\end{array}$ & TDH da tensão $\%$ & $\begin{array}{c}\text { TDH da corrente } \\
\mathbf{\%}\end{array}$ & $\begin{array}{c}\text { Fase da fundamental da corrente } \\
\text { Graus }\end{array}$ \\
\hline $\mathbf{5}$ & 4 & 29 & -0.98 \\
\hline $\mathbf{1 0}$ & 4 & 30 & -3.62 \\
\hline $\mathbf{2 0}$ & 4 & 33 & -4.45 \\
\hline $\mathbf{3 0}$ & 4 & 27 & -5.44 \\
\hline $\mathbf{4 0}$ & 4 & 22 & -6.14 \\
\hline $\mathbf{5 0}$ & 4 & 17 & -6.33 \\
\hline $\mathbf{6 0}$ & 4 & 14 & -6.76 \\
\hline $\mathbf{7 0}$ & 4 & 10 & -8.48 \\
\hline $\mathbf{8 0}$ & 4 & 9 & -8.54 \\
\hline $\mathbf{9 0}$ & 4 & 7 & -8.61 \\
\hline $\mathbf{1 0 0}$ & 4 & 6 & -9.69 \\
\hline
\end{tabular}

A partir das Figuras 12 e 13, observa-se que para $20 \%$ da carga tem-se uma distorção harmônica total de 33\%, correspondendo a um fator de potência de 0,947.

A Figura 14 apresenta a tensão e a corrente no IGBT. A tensão e a corrente de saída são mostradas nas Figuras 15 e 16 respectivamente, e a Figura 17 mostra o comportamento da tensão nos terminais do capacitor $\mathrm{C}_{1}$.

$\mathrm{Na}$ Figura 18 tem-se o rendimento do conversor em função da potência processada.

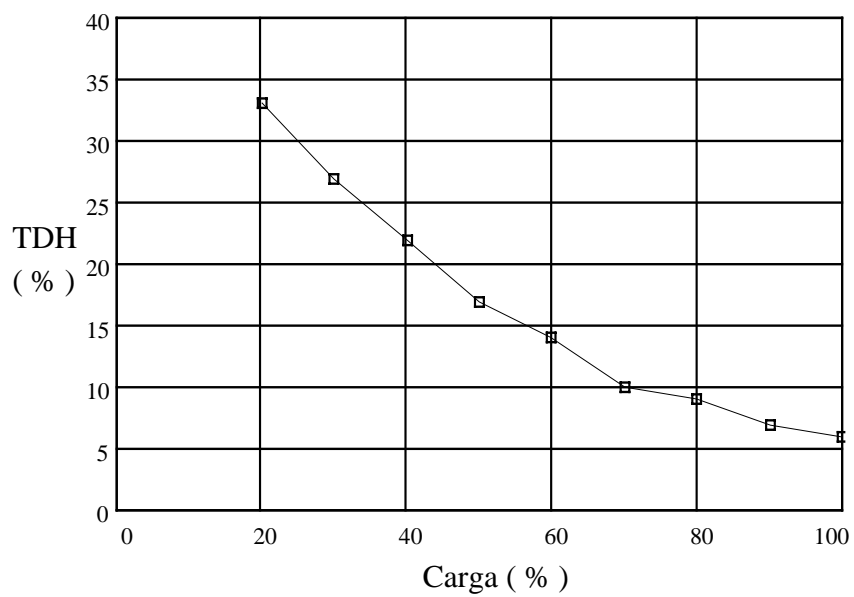

Figura 12 - TDH x Carga.

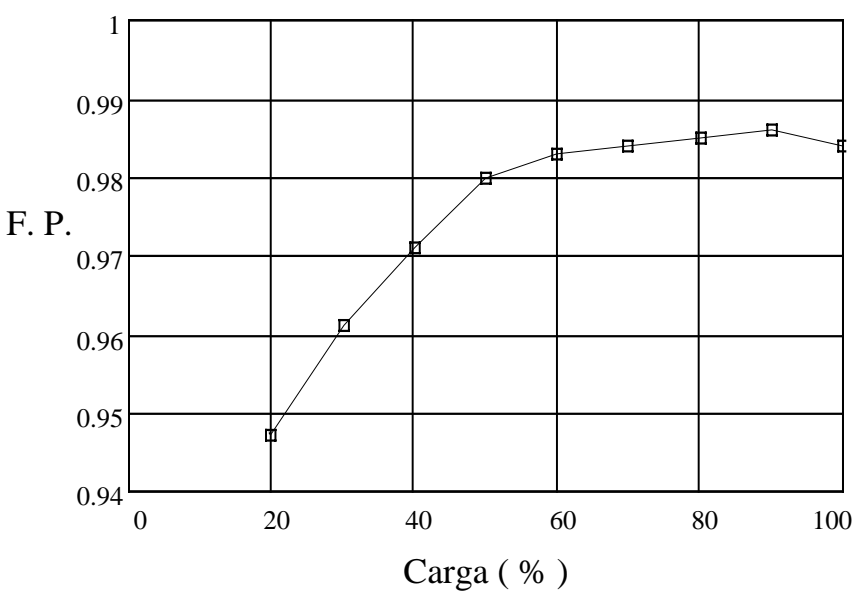

Figura 13 - Fator de potência x Carga.

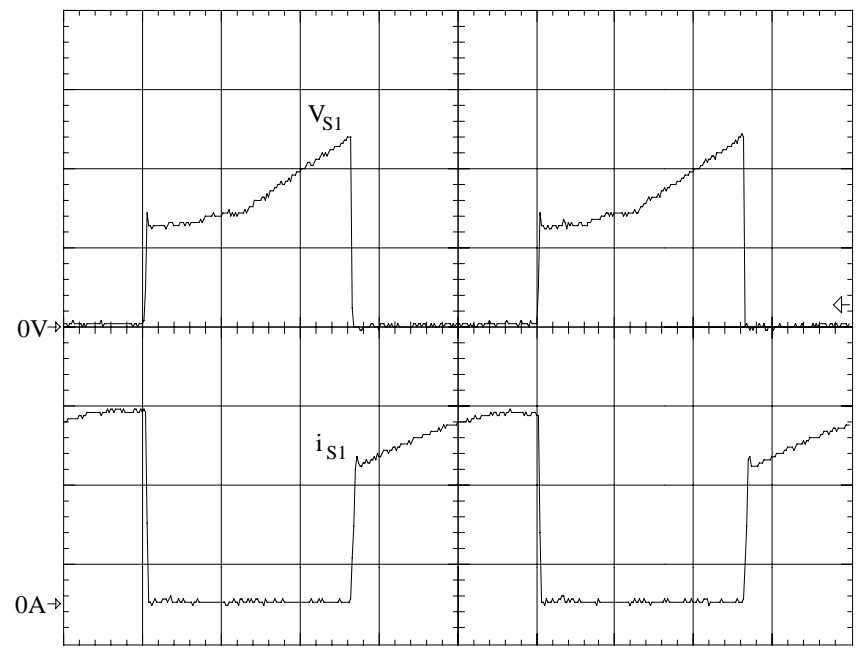

Figura 14 - Tensão e corrente na chave S1. Escalas: $200 \mathrm{~V} / \mathrm{div}, 10 \mathrm{~A} / \mathrm{div}, 10 \mu \mathrm{s} / \mathrm{div}$. 


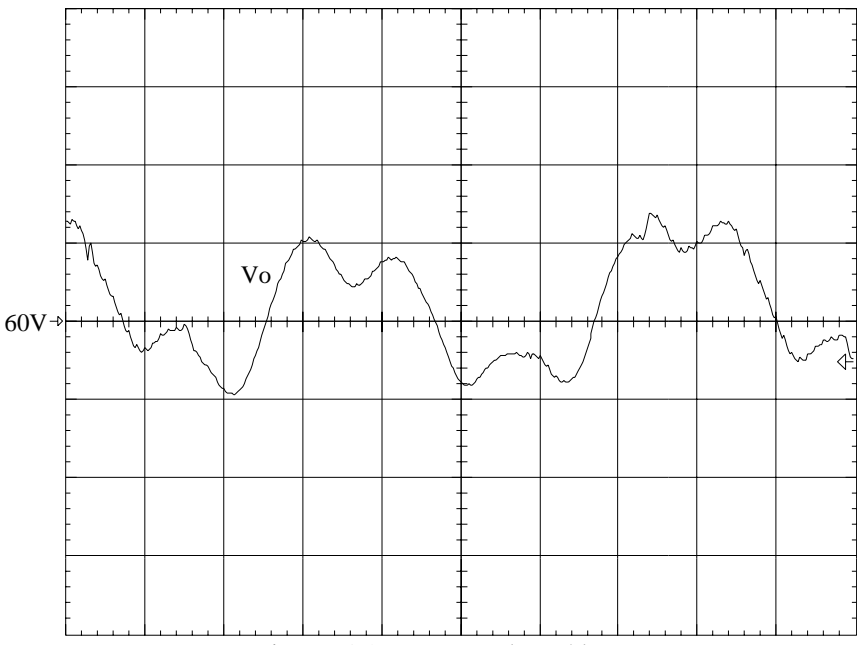

Figura 15 - Tensão de saída. Escalas: $500 \mathrm{mV} / \mathrm{div}, 2 \mathrm{~ms} / \mathrm{div}$.

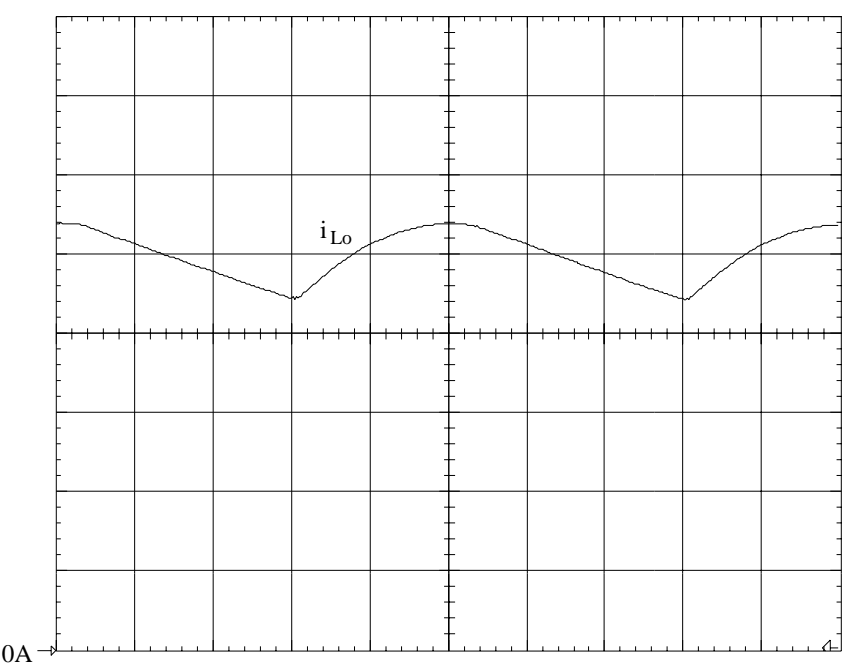

Figura 16 - Corrente no indutor de saída. Escalas: 5A/div, $10 \mu \mathrm{s} / \mathrm{div}$.

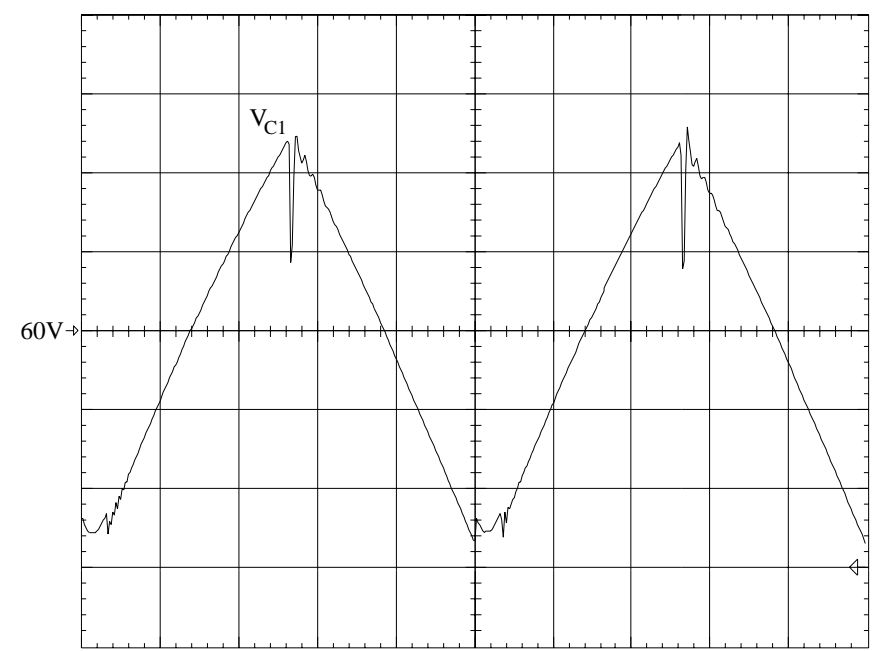

Figura 17 - Tensão no capacitor de acoplamento. Escalas: 2V/div, $10 \mu \mathrm{s} / \mathrm{div}$.

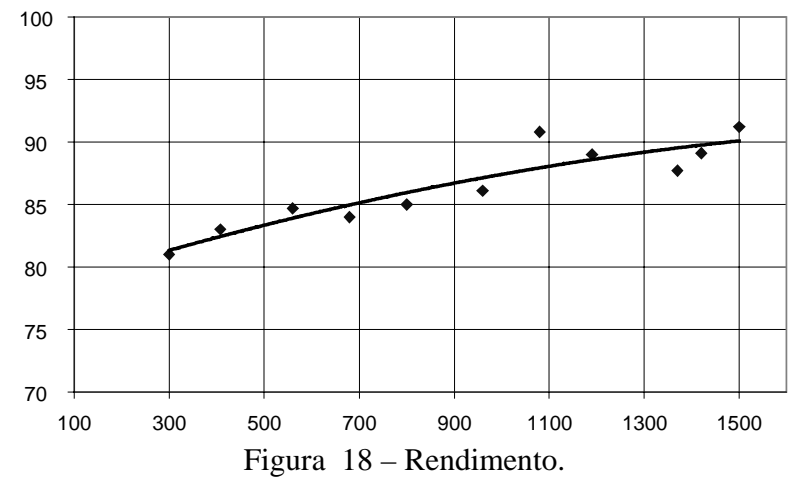

VI. CONCLUSÕES

Este artigo apresentou a análise de um retificador trifásico empregando o conversor CC-CC Zeta, operando no modo de condução contínua, para correção do Fator de Potência.

A utilização do conversor Zeta em condução contínua permite diminuir os picos da corrente na chave principal e nos elementos magnéticos, e conseqüentemente o valor eficaz da corrente nos mesmos. Dessa forma, tem-se uma diminuição das perdas por condução.

De acordo com os resultados obtidos, tem-se um conversor CA-CC com as seguintes características:

- estrutura simples e robusta;

- alto fator de potência com condução contínua; portanto, mais adequado para aplicações em alta potência;

- naturalmente isolado;

- opera tanto como elevador quanto abaixador de tensão;

- permite a regulação da tensão de saída com um único estágio de processamento de energia, e empregando uma única chave controlada;

- em caso de falha a chave pode ser acionada para abrir, protegendo o sistema;

- saída do tipo de fonte de corrente, facilitando a associação de vários módulos em paralelo.

Diante dessas características os autores acreditam que esta estrutura pode ser de muita utilidade para certas aplicações industriais.

\section{REFERÊNCIAS BIBLIOGRÁFICAS}

[1] A.C. Prasad, P.D. Ziogas, S. Manias, "An Active Power Factor Correction Technique For Three-Phase Diode Rectifiers", IEEE-PESC'89, pp. 58-66.

[2] J. He, N. Mohan, C. Wong, "Unity Power Factor ThreePhase Diode Bridge AC-DC Converter with Soft Switchings", PCC-Yokohama, pp. 514-519, 1993.

[3] L. Malasani et alli, "Single-Switch Three-Phase AC-DC Converter with High Power Factor and wide Regulation Capability", IEEE-PESC, pp. 279-285, 1992.

[4] J. W. Kolar, H. Ertl, and F. C. Zach, "A Comprehensive Design Approach For a Three-Phase High-Frequency Single Switch Discontinuous-Mode Boost Power Factor Corrector Based on Analytically Derived Normalized 
Converter Component Ratings", in IEEE/IAS'93 Conf., pp. 931-938.

[5] D. Simonetti, J. Sebastian, and J. Uceda, "Single-Switch Three-Phase Power Factor Preregulator Under Variable Switching Frequency And Discontinuous Input Current", in IEEE Power Electronics Specialists Conf., 1993, pp. 657-662.

[6] O. Huang and F. Lee, "Harmonic Reduction In A Single Switch Three-Phase Boost Rectifier With High Order Harmonic Injected PWM", in IEEE Power Electronics Specialists Conf., 1996, pp. 1266-1271.

[7] K. Schenk and S. Cuk, "A Simple Three-Phase Power Factor Corrector With Improved Harmonic Distortion", in IEEE Power Electronics Specialists Conf., 1997, pp. 399405 .

[8] E. Ismail and R. W. Erickson, "A Single Transistor Three-Phase Resonant Switch For High Quality Rectification", in IEEE Power Electronics Specialists Conf., 1992, pp. 1341-1351.

[9] C. T. Pan and T. C. Chen, "Step-up/down Three-Phase AC to DC Converter with Sinusoidal Input Current and Unit Power Factor", in IEE Proc. Power Electronics Applications, 1994, vol. 141, nº 2, pp. 77-84.

[10]V. Vlatkovic et alli, "Analysis and Design of a ZeroVoltage Switched Three-Phase PWM Rectifier with Power Factor Correction", IEEE-PESC, 1992, pp. 13521360.

[11]E. Ismail and R. W. Erickson, "A New Class of Low Cost Three-Phase High Quality Rectifier with ZeroVoltage Switching”, IEEE-APEC, 1993, pp. 182-189.

[12]J. C. Fagundes, C. M. T. Cruz and I. Barbi, "Active Power Factor Correction in a Three-Phase Rectifier by Switching the AC Line Current", COBEP'93, 1993, pp. 46-51.

[13]M.M. Casaro, "Retificador Trifásico com Alto Fator de Potência Usando o Conversor CC-CC Zeta no Modo de Condução Contínua", Dissertação de Mestrado, INEP/ EEL/UFSC, Florianópolis, SC, 1996.

[14]A. F. de Souza, "Retificadores Monofásicos de Alto Fator de Potência com Reduzidas Perdas de Condução e Comutação Suave", Tese de Doutorado, INEP/EEL/UFSC, Florianópolis, SC, 1998.

\section{DADOS BIOGRÁFICOS}

Denizar Cruz Martins, nasceu em São Paulo - São Paulo, em 24 de Abril de 1955. Formou-se em Engenharia Elétrica e obteve o título de Mestre em Engenharia Elétrica pela Universidade Federal de Santa Catarina, Florianópolis - SC em 1978 e 1981, respectivamente. Concluiu o Doutorado no INPT, Toulouse - França, em 1986. Atualmente é professor titular do Depto. de Engenharia Elétrica da Universidade Federal de Santa Catarina, Florianópolis - SC. O Prof. Denizar já realizou mais de 30 consultorias técnicas e obteve 02 patentes de invenção. Sua área de atuação compreende: desenvolvimento de conversores para tratamento de energia solar e simulação de Conversor Estáticos. É membro da SOBRAEP, da SBA e do IEEE.

Marcio Mendes Casaro, Nasceu em Assis, São Paulo, em 5 de maio de 1972. Concluiu o curso de Engenharia Elétrica pela Escola de Engenharia de Lins, Lins, São Paulo, em 1994 e obteve o grau de Mestre em Engenharia Elétrica pela Universidade Federal de Santa Catarina em 1996. Atualmente atua como professor no Curso Superior de Tecnologia em Eletrônica na modalidade Automação de Processos Industriais, pelo Centro Federal de Educação Tecnológica do Paraná, Ponta Grossa, Paraná. Sua área de interesse inclui aplicações em Eletrônica de Potência, em especial, fontes de alimentação com correção de fator de potência.

Ivo Barbi, nasceu em Gaspar (SC), em 1949. Formou-se em Engenharia Elétrica pela Universidade Federal de Santa Catarina - UFSC, em 1973. Em 1976 recebeu o título de Mestre pela mesma Universidade e em 1979 recebeu o título de Doutor pelo Institut National Polytechnique de Toulouse, França. Desde 1974 é professor da UFSC e atualmente professor titular do Departamento de Engenharia Elétrica. É membro fundador da SOBRAEP tendo sido seu primeiro presidente. Desde 1992, é Editor Associado na área de Conversores de Potência da IEEE Transactions on Industrial Electronics. Suas áreas de atuação compreendem modelagem, análise, projeto e aplicações de conversores estáticos operando em alta frequiência e correção de fator de potência de fontes de alimentação. 